\title{
铜催化烯醚的三氟甲基化反应
}

\author{
彭 芯 ${ }^{a}$ 杨涁沝 ${ }^{a} \quad$ 陈志敏 ${ }^{a}$ 徐 力 ${ }^{b}$ 王少华 $*, a, b$ \\ $\left({ }^{a}\right.$ 兰州大学化学化工学院 兰州 730000) \\ $\left({ }^{b}\right.$ 兰州大学药学院 兰州 730000)
}

\begin{abstract}
摘要 在澳化亚铜和醋酸亚铜的共同作用下，成功实现了烯梄的三氟甲基化反应. 该反应为 2 -三氟甲基烯梄类化合物 的合成提供了一条潜在的合成途径. 该反应条件温和, 收率良好, 并且具有很好的官能团容忍性. 产物结构经 NMR, IR, MS 及 HRMS 数据得以证实.
\end{abstract}

关键词＼cjkstart铜；氟; 烯梄; 三氟甲基

\section{Copper-Catalyzed Trifluoromethylation of Enol Ether}

\author{
Peng, Rui ${ }^{a} \quad$ Yang, Binmiao $^{a} \quad$ Chen, Zhimin $^{a} \quad \mathrm{Xu}^{a} \mathrm{Li}^{b} \quad$ Wang, Shaohua ${ }^{\text {* }, a, b}$ \\ $\left({ }^{a}\right.$ College of Chemistry and Chemical Engineering, Lanzhou University, Lanzhou 730000) \\ $\left({ }^{b}\right.$ School of Pharmacy, Lanzhou University, Lanzhou 730000)
}

\begin{abstract}
A copper-catalyzed trifluoromethylation of enol ether is developed, which provides a potential strategy for the synthesis of 2-trifluoromethyl enol ether compounds. The reaction is under mild conditions with moderate to good yields. The structures of target compounds were confirmed by NMR, IR, MS, and HRMS.

Keywords copper; fluorine; enol ether; trifluoromethyl
\end{abstract}

有机氟化合物由于单个或多个氟原子的引入, 使得 它们会表现出一些非常独特的物理、化学性质及特殊的 生物活性, 因此, 相关领域的研究一直以来都是化学家 们的关注的重点内容之一. 随着相关研究的不断深入, 有机氟化学也成为有机化学一个非常重要的分支. 其 中, 作为含氟有机化合物的一类特殊代表, 三氟甲基取 代的有机化合物, 由于强吸电子性三氟甲基的存在, 使 得它们通常会表现出一定的代谢稳定性和亲脂性, 因 而, 该类化合物在农业、药学和功能材料上都有着重要 的用途 ${ }^{[1]}$. 相应的, 发展高效、简捷的 $\mathrm{C}-\mathrm{CF}_{3}$ 键构筑的 有机合成方法在近些年来受到了有机合成化学家们的 广泛关注. 但是, 传统的 $\mathrm{C}-\mathrm{CF}_{3}$ 键的构筑方法大都需 要比较苛刻的反应条件, 对于常见的官能团的耐受性较 差, 因而相关方法的具体应用范围非常有限 ${ }^{[2]}$; 而过渡 金属催化的三氟甲基化反应，由于金属催化剂的存在， 在比较温和的反应条件下一般能够给出比较好的底物 适用范围, 很大程度上能弥补传统方法的不足 ${ }^{[3]}$. 在常
用的过渡金属催化剂中，金属铜因其廉价、活性较高等 优点, 近几年被广泛地用于相关的催化三氟甲基化反 应. 并且, 相应的底物适用范围较广, 其中对于卤代 烃 ${ }^{[4]}$ 、有机硼试剂 ${ }^{[5]}$ 、烷烃 ${ }^{[6]}$, 、烯烃 ${ }^{[7]}$ 和炔烃 ${ }^{[8]}$ 的三氟 甲基化等都十分有效. 但是, 对于烯醚这一类特殊的有 机分子，在相应的碳一碳双键以及烯丙位进行选择性的 三氟甲基化反应的研究, 到目前为止仍很少有见报道 ${ }^{[9]}$. 因此，本文以烯醚分子中碳一碳双键的选择性三氟甲基 化为研究目标, 在铜的催化下成功实现了相应分子的选 择性 $\mathrm{C}_{\mathrm{sp} 2}-\mathrm{CF}_{3}$ 键的构筑(Eq. 1).

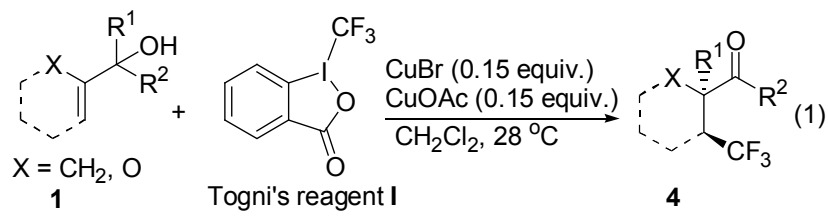

\section{1 结果与讨论}

2013 年, 我们成功地发展了一类铜催化的三氟甲

* E-mail: wangshh@1zu.edu.cn

Received March 17, 2014; revised April 10, 2014; published online April 16, 2014.

Project supported by the National Natural Science Foundation of China (No. 21202073) and the Fundamental Research Funds for the Central Universities (No. lzujbky-2012-216).

国家自然科学基金(No. 21202073)和兰州大学中央高校专项基金(No. 1zujbky-2012-216)资助项目. 
基化/半频哪醇重排的串联反应(Eq. 1), 该方法为 $\beta$-三氟 甲基羰基化合物的合成提供了一个非常有效的途径. 在 后续对反应机理的研究过程中我们推测, 底物 1 与三氟 甲基试剂反应后可能会存在中间体 $\mathbf{3}$, 相应的中间体 $\mathbf{3}$ 可以接着发生 1,2 迁移重排反应生成之前报道的三氟甲 基化/半片那醇重排反应的产物 4, 而另一方面, 如果中 间体 3 发生氢消除反应，则可以得到 2-三氟甲基烯醚化 合物 2. 而要成功地实现相应的反应过程的选择性控制, 得到化合物 $\mathbf{2}$ 的同时抑制化合物 $\mathbf{4}$ 的生成可以通过两方 面的调节来实现: 第一, 降低迁移基团的迁移动力; 第 二, 通过改变底物的环系结构, 这样有可能增强氢消除 的动力 (Scheme 1).
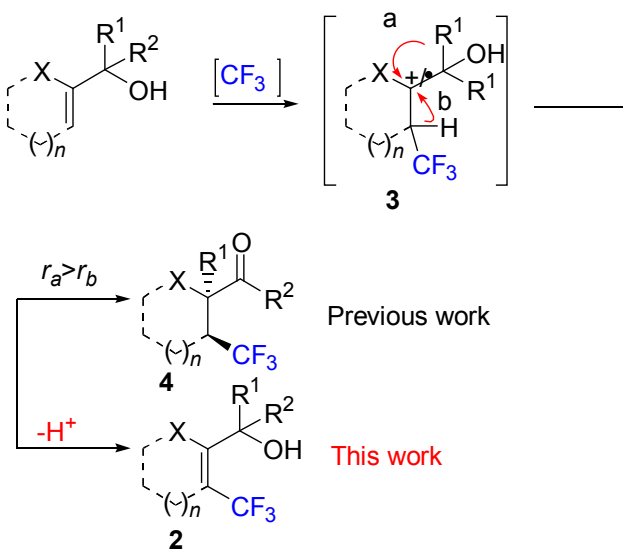

\section{Scheme 1}

在这种思想的指导下, 我们以化合物 $1 \mathrm{a}$ 为模拟底 物, 对反应的条件进行了篮选. 与设想的一样, 在 CuOTf 的催化下, 化合物 1a 可以与三氟甲基化试剂 I 顺利地发生反应，以 $20 \%$ 的收率得到了预期的产物 $2 \mathrm{a}$. 通过对催化剂的进一步篎选, 通过 $\mathrm{CuBr}$ 或者 $\mathrm{CuOAc}$ 的 使用可以将反应的产率进一步分别优化到 $68 \%$ 和 $66 \%$. 此外，我们还发现，不同的三氟甲基化试剂对于反应产 率的影响也十分明显. 在相同的反应条件下, 使用试 剂 II 作为三氟甲基的供体时，反应的产率下降为 40\%; 试剂 III 的使用只能给出衡量的预期产物, 大部分的原 料都随着反应的进行而逐渐分解. 同时, 该反应的溶剂 效应也十分明显, 使用乙腈作溶剂时, 反应的产率下降 为 $12 \%$; 而当使用极性质子性溶剂甲醇时, 只能得到衡 量的产物 2a. 十分有趣的是, $\mathrm{CuBr}$ 和 $\mathrm{CuOAc}$ 的混合使 用可以将反应的收率进一步优化到 $80 \%$.

在对反应条件进行优化之后, 我们分别合成了迁移 动力较差的 $1 \mathrm{~b}$, 以及二氢呋喃环底物 $1 \mathrm{c}$ 和 $1 \mathrm{~d}$. 与我们 所预想的一样, 所合成的四个底物在溴化亚铜和醋酸亚 铜的共同作用下, 都很顺利地发生了设计的烯烃三氟甲 基化反应，而没有发生重排反应. 同时, 实现烯醚双键
表 1 三氟甲基化反应的优化过程 ${ }^{a}$

Table 1 Optimization of reaction conditions of the trifluoromethylation of enol ether<smiles>OC(C1CCCCC1)(C1CCCCC1)C1CCCCO1</smiles>

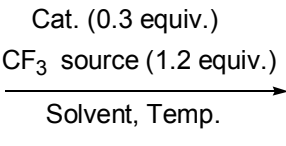<smiles>OC(C1=C(C(F)(F)F)CCCO1)(C1CCCCC1)C1CCCCC1</smiles>

2a

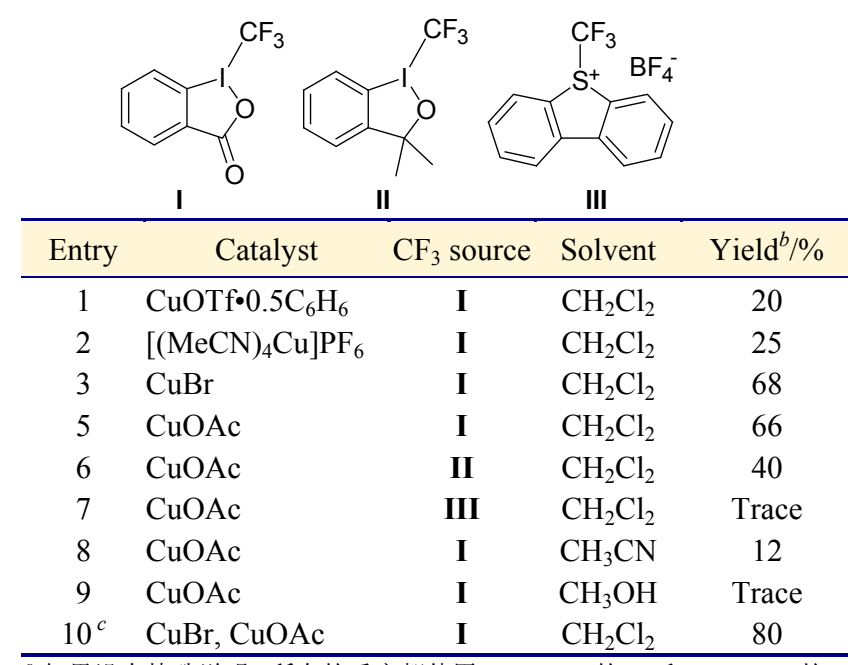

${ }^{a}$ 如果没有特殊说明, 所有的反应都使用 $0.1 \mathrm{mmol}$ 的 $\mathbf{1 a}$ 和 1.2 equiv.的三 氟甲基化试剂在 $28{ }^{\circ} \mathrm{C}$ 下进行反应; ${ }^{b}$ 分离产率; ${ }^{c}$ 用 $0.015 \mathrm{mmol}$ 的 $\mathrm{CuOAc}$ 和 $0.015 \mathrm{mmol} \mathrm{CuBr}$ 作为催化剂.

表 2 铜催化的烯醚双键的三氟甲基化

Table 2 Scope of the trifluoromethylation of enol ether by copper(I)

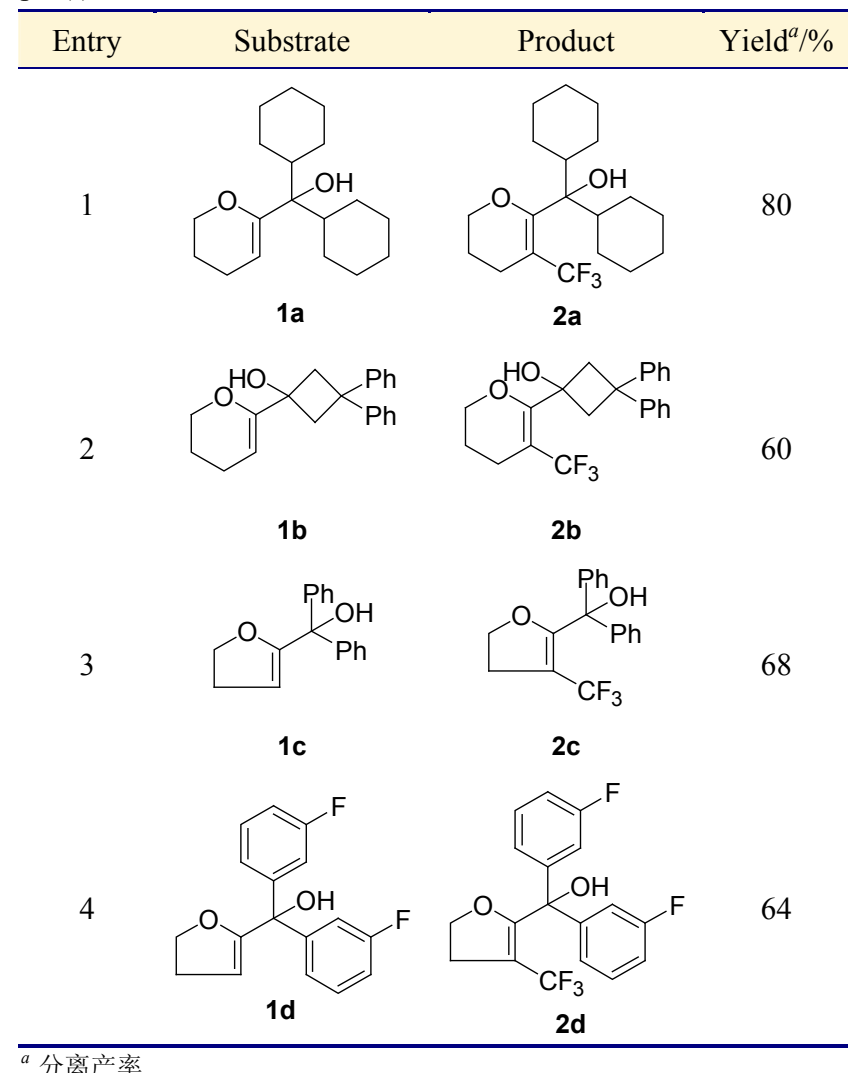

$a$ 分离产率. 
的三氟甲基化构筑 $\mathrm{C}_{\mathrm{sp} 2}-\mathrm{CF}_{3}$ 键的同时，该反应很好的 保留了底物分子原有的官能团, 反应条件温和. 虽然烯 胺化合物的三氟甲基化已经有报道, 但是就我们所了 解, 本文所介绍的烯醚化合物的三氟甲基化反应为该类 反应的首次报道，该个反应为 2 -三氟甲基烯醚类化合物 的合成提供了一条潜在的合成途径.

\section{2 结论}

我们以溴化亚铜和醋酸亚铜作为催化剂, 二氯甲烷 作为溶剂的条件下, 以 Togni's reagent $\mathrm{I}$ 作为三氟甲基 化试剂，对烯醚进行三氟甲基化构筑 $\mathrm{C}_{\mathrm{sp}^{2}}-\mathrm{CF}_{3}$ 键，该 反应条件温和, 产率良好, 官能团容忍性强, 不会对底 物的双键和羟基造成影响, 为化合物的后续衍生保留的 相应的官能团.

\section{3 实验部分}

\section{1 仪器与试剂}

实验所用溶剂使用前均按照处理溶剂的标准方法 在氩气环境下进行处理; ${ }^{1} \mathrm{H},{ }^{13} \mathrm{C}$ 以及 ${ }^{19} \mathrm{~F}$ 核磁谱图均在 Bruker AX-400 MHz 仪器中测定, $\mathrm{CDCl}_{3}$ 为溶剂, $\mathrm{TMS}$ 为 内标; 红外光谱用 Nicolet FT-170SX 型红外光谱仪 $(\mathrm{KBr}$ 压片); MS 采用岛津 2010 型质谱仪型仪器测定; 高分辨 率质谱用 Bruker ApexII 通过 ESI 源测定.

\section{2 实验方法}

3.2.1 二环已基 (3,4-二氢- $2 H$-吡喃-6-烯)甲醇(1a)的 合成

将 3,4-二氢-2H-吡喃(1.84 g, $21.9 \mathrm{mmol}$ )溶于 $60 \mathrm{~mL}$ 干燥四氢呋喃中, 体系在氩气保护下, 于 $0{ }^{\circ} \mathrm{C}$ 下缓慢加 入正丁基锂 $(2.5 \mathrm{~mol} / \mathrm{L}$ in hexane, $8.0 \mathrm{~mL}, 20.0 \mathrm{mmol})$, 加 完后将体系转入室温反应 $3 \mathrm{~h}$. 体系冷却至 $-78{ }^{\circ} \mathrm{C}$, 于 氩气保护下将二环已基甲酮 $(1.94 \mathrm{~g}, 21.9 \mathrm{mmol}$ )加入反 应体系, 加完后体系转移到室温环境摚拌 $2 \mathrm{~h}$. 体系用 饱和碳酸氢钠溶液 $(15 \mathrm{~mL})$ 淬灭, 分离有机相, 水相用乙 醚 $(30 \mathrm{~mL} \times 2)$ 萃取, 合并有机相, 有机相用饱和氯化钠 溶液洗, 无水硫酸钠干燥, 过滤, 滤液减压浓缩. 粗产 物用硅胶桂层析 $[V$ (petroleum) $: V(\mathrm{EtOAc})=110: 1]$, 得 到白色无定型固体 (83\%产率). ${ }^{1} \mathrm{H}$ NMR $(400 \mathrm{MHz}$, $\left.\mathrm{CDCl}_{3}\right) \delta: 4.70(\mathrm{t}, J=3.8 \mathrm{~Hz}, 1 \mathrm{H}), 3.92(\mathrm{t}, J=5.0 \mathrm{~Hz}, 1 \mathrm{H})$, $2.06 \sim 2.02(\mathrm{~m}, 2 \mathrm{H}), 1.77 \sim 1.70(\mathrm{~m}, 9 \mathrm{H}), 1.65 \sim 1.57(\mathrm{~m}$, $6 \mathrm{H}), 1.26 \sim 1.02(\mathrm{~m}, 10 \mathrm{H}) ;{ }^{13} \mathrm{C} \mathrm{NMR}\left(100 \mathrm{MHz}, \mathrm{CDCl}_{3}\right) \delta$ : 155.0, 95.6, 79.3, 65.6, 42.1, 27.4, 27.0, 26.8, 26.6, 22.5, 20.0; IR (neat) $v: 3492,2929,1448,1065 \mathrm{~cm}^{-1}$; MS (EI) m/z (\%): 278 (6), 195 (100), 177 (15), 111 (25), 83 (40), 55 (77); HRMS (ESI) calcd for $\mathrm{C}_{18} \mathrm{H}_{31} \mathrm{O}_{2}(\mathrm{M}+\mathrm{H})^{+}: 279.2319$, found 279.2322 .
3.2.2 1-(3,4-二氢- $2 H$-吡喃-6-烯)-3,3-二苯基环丁醇 (1b) 的合成

实验操作与 $1 \mathrm{a}$ 的合成类似, 粗产物用硅胶桂层析 $[V($ petroleum $): V(\mathrm{EtOAc})=60: 1]$, 得到白色无定型固 体(84\%产率). ${ }^{1} \mathrm{H}$ NMR (400 MHz, $\mathrm{CDCl}_{3}$ ) $\delta: 7.34 \sim 7.32$ $(\mathrm{m}, 2 \mathrm{H}), 7.28 \sim 7.21(\mathrm{~m}, 6 \mathrm{H}), 7.12 \sim 7.06(\mathrm{~m}, 2 \mathrm{H}), 4.65(\mathrm{t}$, $J=3.8 \mathrm{~Hz}, 1 \mathrm{H}), 3.92$ (t, $J=5.2 \mathrm{~Hz}, 2 \mathrm{H}), 3.32$ (d, $J=2.4$ $\mathrm{Hz}, 1 \mathrm{H}), 3.29$ (d, $J=2.8 \mathrm{~Hz}, 1 \mathrm{H}), 2.94(\mathrm{~s}, 1 \mathrm{H}), 2.91$ (s, $1 \mathrm{H}), 2.32(\mathrm{~s}, 1 \mathrm{H}), 1.87 \sim 1.83(\mathrm{~m}, 2 \mathrm{H}), 1.70 \sim 1.64(\mathrm{~m}$, $2 \mathrm{H}) ;{ }^{13} \mathrm{C}$ NMR $\left(100 \mathrm{MHz}, \mathrm{CDCl}_{3}\right) \delta: 155.1,150.1,149.0$, 128.3, 128.0, 126.4, 126.2, 125.5, 125.3, 96.0, 71.4, 66.3, 46.6, 43.4, 22.0, 19.8; IR (neat) $v: 3442,2945,1087,739$, $702 \mathrm{~cm}^{-1}$; MS (EI) $\mathrm{m} / z$ (\%): 306 (40), 180 (88), 165 (72), 126 (100), 98 (58), 55 (47); HRMS (ESI) calcd for $\mathrm{C}_{21} \mathrm{H}_{23} \mathrm{O}_{2}(\mathrm{M}+\mathrm{H})^{+}:$307.1693, found 307.1688.

$3.2 .3(4,5$-二氢呋喃-2-烯) 二(3-氟苯) 甲醇 $(1 \mathrm{~d})$ 的合成 实验操作与 $1 \mathrm{a}$ 的合成类似, 得到粗产物用硅胶桂 层析 $[V$ (petroleum) $: V($ EtOAc $)=80: 1]$, 得到白色无定 型固体(97\%产率). ${ }^{1} \mathrm{H}$ NMR (400 MHz, $\left.\mathrm{CDCl}_{3}\right) \delta: 7.30 \sim$ $7.24(\mathrm{~m}, 2 \mathrm{H}), 7.17 \sim 7.14(\mathrm{~m}, 4 \mathrm{H}), 7.00 \sim 6.94(\mathrm{~m}, 2 \mathrm{H})$, $4.57(\mathrm{t}, J=3.0 \mathrm{~Hz}, 1 \mathrm{H}), 4.45(\mathrm{t}, J=9.2 \mathrm{~Hz}, 2 \mathrm{H}), 3.26(\mathrm{~s}$, $1 \mathrm{H}), 2.70(\mathrm{dt}, J=2.4 \mathrm{~Hz}, 9.2 \mathrm{~Hz}, 2 \mathrm{H}) ;{ }^{13} \mathrm{C}$ NMR $(100$ $\left.\mathrm{MHz}, \mathrm{CDCl}_{3}\right) \delta: 163.8(\mathrm{~d}, J=468.0 \mathrm{~Hz}), 161.4,159.1$, 146.0 (d, $J=7.0 \mathrm{~Hz}), 129.4$ (d, $J=8.0 \mathrm{~Hz}), 122.7$ (d, $J=$ $3.0 \mathrm{~Hz}), 114.6(\mathrm{~d}, J=21.0 \mathrm{~Hz}), 114.2(\mathrm{~d}, J=23.0 \mathrm{~Hz})$, 100.8, 76.8, 70.9, 30.2; IR (neat) $v: 3477,2926,1590$, 1443, 1228, 781, $697 \mathrm{~cm}^{-1}$; MS (EI) $\mathrm{m} / z$ (\%): 288 (10), 259 (25), 165 (23), 123 (100), 95 (28); HRMS (ESI) calcd for $\mathrm{C}_{17} \mathrm{H}_{15} \mathrm{~F}_{2} \mathrm{O}_{2}(\mathrm{M}+\mathrm{H})^{+}: 289.1035$, found 289.1030 .

3.2.4 二环已基 [(5-(三氟甲基)-3,4-二氢- $2 H$-吡喃-6烯]甲醇 (2a) 的合成

将溴化亚铜 $(0.015 \mathrm{mmol})$, 醋酸亚铜 $(0.015 \mathrm{mmol})$ 和 Togni 试剂 $\mathbf{I}(0.15 \mathrm{mmol})$ 加入二氯甲烷 $(1.0 \mathrm{~mL})$ 中在氩 气保护下于室温环境中摚拌 $10 \mathrm{~min}$, 将 $\mathbf{1 a}(0.10 \mathrm{mmol})$ 的二氯甲烷 $(0.5 \mathrm{~mL})$ 溶液加入反应体系中于 $28{ }^{\circ} \mathrm{C}$ 下反 应. 薄层色谱监测, 当 $1 \mathrm{a}$ 完全消失时反应体系直接用硅 胶柱层析 $\left[V\right.$ (petroleum)： $\left.V\left(\mathrm{CH}_{2} \mathrm{Cl}_{2}\right)=4: 1\right]$ 得到白色晶 状固体 2a (27.8 mg, 80\%产率). m.p. 99 $101{ }^{\circ} \mathrm{C} ;{ }^{1} \mathrm{H}$ NMR $\left(400 \mathrm{MHz}, \mathrm{CDCl}_{3}\right) \delta: 3.91(\mathrm{t}, J=4.2 \mathrm{~Hz}, 2 \mathrm{H})$, $2.28 \sim 2.25(\mathrm{~m}, 2 \mathrm{H}), 2.03(\mathrm{~s}, 1 \mathrm{H}), 1.86 \sim 1.58(\mathrm{~m}, 14 \mathrm{H})$, $1.30 \sim 0.99(\mathrm{~m}, 10 \mathrm{H}) ;{ }^{13} \mathrm{C}$ NMR $\left(100 \mathrm{MHz}, \mathrm{CDCl}_{3}\right) \delta$ : $162.2\left(\mathrm{~d}, J_{\mathrm{F}}=4.0 \mathrm{~Hz}\right), 126.1\left(\mathrm{q}, J_{\mathrm{F}}=269.0 \mathrm{~Hz}\right), 101.9(\mathrm{q}$, $\left.J_{\mathrm{F}}=32.0 \mathrm{~Hz}\right), 81.9,64.9,43.7,27.9,27.1,26.7\left(\mathrm{~d}, J_{\mathrm{F}}=\right.$ $10.0 \mathrm{~Hz}), 22.1\left(\mathrm{~d}, J_{\mathrm{F}}=4.0 \mathrm{~Hz}\right), 21.6 ;{ }^{19} \mathrm{~F}$ NMR $(376 \mathrm{MHz}$, $\left.\mathrm{CDCl}_{3}\right) \delta:-49.9$; IR (neat) $v: 3641,2928,1629,1450$, 
1268, $802 \mathrm{~cm}^{-1}$; MS (EI) $\mathrm{m} / \mathrm{z}$ (\%): 346 (6), 263 (63), 243 (90), 223 (50), 161 (52), 55 (100); HRMS (ESI) calcd for $\mathrm{C}_{19} \mathrm{H}_{30} \mathrm{~F}_{3} \mathrm{O}_{2}(\mathrm{M}+\mathrm{H})^{+}: 347.2192$, found 347.2186.

3.2.5 3,3-二苯基-1-[5-(三绨甲基)-3,4-二氢- $2 H$ - 吡 喃-6-烯]环丁醇 $(2 b)$ 的合成

实验操作与 2a 的合成类似, 体系用硅胶柱层析 [ $V$ (petroleum) $: V\left(\mathrm{CH}_{2} \mathrm{Cl}_{2}\right)=1: 1$ ] 得到无色油状产物 (22.4 mg, 60\%产率). ${ }^{1} \mathrm{H}$ NMR (400 $\mathrm{MHz}, \mathrm{CDCl}_{3}$ ) $\delta$ : $7.37 \sim 7.35(\mathrm{~m}, 2 \mathrm{H}), 7.29 \sim 7.22(\mathrm{~m}, 6 \mathrm{H}), 7.13 \sim 7.08(\mathrm{~m}$, $2 \mathrm{H}), 3.84$ (t, $J=5.0 \mathrm{~Hz}, 2 \mathrm{H}), 3.46(\mathrm{~d}, J=13.2 \mathrm{~Hz}, 2 \mathrm{H})$, 3.05 (d, $J=13.2 \mathrm{~Hz}, 2 \mathrm{H}), 2.17$ (s, 1H), 2.09 (t, $J=6.4 \mathrm{~Hz}$, $2 \mathrm{H}), 1.64 \sim 1.58(\mathrm{~m}, 2 \mathrm{H}) ;{ }^{13} \mathrm{C} \mathrm{NMR}\left(100 \mathrm{MHz}, \mathrm{CDCl}_{3}\right) \delta$ : $160.3\left(\mathrm{~d}, J_{\mathrm{F}}=4.0 \mathrm{~Hz}\right), 149.2,149.0,128.4,128.1,126.3$, $126.1,125.6,125.5,125.5\left(\mathrm{q}, J_{\mathrm{F}}=268.0 \mathrm{~Hz}\right), 99.2\left(\mathrm{~d}, J_{\mathrm{F}}=\right.$ $32.0 \mathrm{~Hz}), 72.766 .3,47.0,43.3,20.7,20.2 ;{ }^{19} \mathrm{~F}$ NMR (376 $\left.\mathrm{MHz}, \mathrm{CDCl}_{3}\right) \delta:-58.7$; IR (neat) v: 3446, 2954, 1648, 1317, 1097, $702 \mathrm{~cm}^{-1}$; MS (EI) $\mathrm{m} / z$ (\%): 374 (10), 356 (5), 180 (100), 165 (40); HRMS (ESI) calcd for $\mathrm{C}_{22} \mathrm{H}_{22} \mathrm{~F}_{3} \mathrm{O}_{2}$ $(\mathrm{M}+\mathrm{H})^{+}: 375.1566$, found 375.1557 .

\subsection{6 二苯基 [3-(三氟甲基)-4,5-二氢呋喃-2-烯]甲醇} (2c) 的合成

实验操作与 $2 \mathrm{a}$ 的合成类似, 体系用硅胶柱层析 $[V($ petroleum $): V($ EtOAc $)=125: 1 \sim 60: 1]$ 得到无色油 状液体(21.8 mg, 68\%产率). ${ }^{1} \mathrm{H}$ NMR $\left(400 \mathrm{MHz}, \mathrm{CDCl}_{3}\right)$ $\delta: 7.45 \sim 7.38(\mathrm{~m}, 10 \mathrm{H}), 4.43(\mathrm{t}, J=9.6 \mathrm{~Hz}, 2 \mathrm{H}), 3.05(\mathrm{t}$, $J=9.6 \mathrm{~Hz}, 2 \mathrm{H}), 2.95(\mathrm{~s}, 1 \mathrm{H}) ;{ }^{13} \mathrm{C} \mathrm{NMR}\left(100 \mathrm{MHz}, \mathrm{CDCl}_{3}\right)$ $\delta: 163.2\left(\mathrm{q}, J_{\mathrm{F}}=5.0 \mathrm{~Hz}\right), 143.2,128.0\left(\mathrm{~d}, J_{\mathrm{F}}=4.0 \mathrm{~Hz}\right), 12$ $7.5,123.9\left(\mathrm{q}, J_{\mathrm{F}}=264.0 \mathrm{~Hz}\right), 78.9,69.3,31.1(\mathrm{~d}, J=2.0$ $\mathrm{Hz}) ;{ }^{19} \mathrm{~F}$ NMR $\left(376 \mathrm{MHz}, \mathrm{CDCl}_{3}\right) \delta$ : -52.1 ; IR (neat) $v$ : $3570,2926,1664,1352,1119,756,701 \mathrm{~cm}^{-1}$; MS (EI) $\mathrm{m} / \mathrm{z}$ (\%): 320 (13), 303 (10), 290 (18), 105 (100), 77 (50); HRMS (ESI) calcd for $\mathrm{C}_{18} \mathrm{H}_{16} \mathrm{~F}_{3} \mathrm{O}_{2}(\mathrm{M}+\mathrm{H})^{+}$: 321.1097 , found 321.1093 .

\subsection{7 双(3-氟苯) [3-(三氟甲基)-4,5-二氢呋喃-2-烯]} 甲醇 $(2 d)$ 的合成

实验操作与 $2 \mathrm{a}$ 的合成类似, 体系用硅胶柱层析 $[V($ petroleum $): V($ EtOAc $)=100: 1 \sim 60: 1]$ 得到无色油 状液体(64\% yield). ${ }^{1} \mathrm{H}$ NMR (400 MHz, $\left.\mathrm{CDCl}_{3}\right) \delta: 7.36 \sim$ $7.30(\mathrm{~m}, 2 \mathrm{H}), 7.17 \sim 7.07(\mathrm{~m}, 4 \mathrm{H}), 7.05 \sim 7.03(\mathrm{~m}, 2 \mathrm{H})$, $4.44(\mathrm{t}, J=9.6 \mathrm{~Hz}, 2 \mathrm{H}), 3.08 \sim 3.00(\mathrm{~m}, 3 \mathrm{H}) ;{ }^{13} \mathrm{C} \mathrm{NMR}$ $\left(100 \mathrm{MHz}, \mathrm{CDCl}_{3}\right) \delta: 163.7\left(\mathrm{~d}, J_{\mathrm{F}}=245.0 \mathrm{~Hz}\right), 162.0(\mathrm{~d}$, $\left.J_{\mathrm{F}}=4.0 \mathrm{~Hz}\right), 161.3,145.1\left(\mathrm{~d}, J_{\mathrm{F}}=7.0 \mathrm{~Hz}\right), 129.6\left(\mathrm{~d}, J_{\mathrm{F}}=\right.$ $8.0 \mathrm{~Hz}), 123.6\left(\mathrm{q}, J_{\mathrm{F}}=265.0 \mathrm{~Hz}\right), 123.0\left(\mathrm{~d}, J_{\mathrm{F}}=3.0 \mathrm{~Hz}\right)$, $115.2\left(\mathrm{~d}, J_{\mathrm{F}}=21.0 \mathrm{~Hz}\right), 114.7\left(\mathrm{~d}, J_{\mathrm{F}}=24.0 \mathrm{~Hz}\right), 102.7(\mathrm{q}$, $\left.J_{\mathrm{F}}=37.0 \mathrm{~Hz}\right), 78.1,69.5,31.0 ;{ }^{19} \mathrm{~F}$ NMR $(376 \mathrm{MHz}$,
$\left.\mathrm{CDCl}_{3}\right) \delta:-52.4,-112.5$; IR (neat) v: 3608, 2926, 1667, 1591, 1485, 1443, 1350, 1123, $783 \mathrm{~cm}^{-1}$; MS (EI) m/z (\%): 356 (4), 123 (69), 95 (56), 57 (100); HRMS (ESI) calcd for $\mathrm{C}_{18} \mathrm{H}_{14} \mathrm{~F}_{5} \mathrm{O}_{2}(\mathrm{M}+\mathrm{H})^{+}: 357.0903$, found 357.0908 .

\section{References}

[1] (a) Uneyama, K. Organofluorine Chemistry, Blackwell, Oxford, U. K., 2006.

(b) Hird, M. Chem. Soc. Rev. 2007, 36, 2070.

(c) Kirk, K. L. Org. Process Res. Dev. 2008, 12, 305.

(d) O'Hagan, D. Chem. Soc. Rev. 2008, 37, 308.

(e) Ojima, I. Fluorine in Medicinal Chemistry and Chemical Biology, Wiley-Blackwell, Chichester, U. K., 2009.

(f) Filler, R; Saha, R. Future Med.Chem. 2009, 1, 777.

(g) Daniels, S.; Tohid, S. F. M.; Velanguparackel, W.; Westwell, A. D. Expert. Opin. Drug Discovery 2010, 5, 291.

(h) Acena, J. L.; Simon-Fuentes, A.; Fustero, S. Curr. Org. Chem. 2010, 14, 928 .

(i) Wang, X.; Zhang, Y.; Wang, J. Sci. China Chem. 2012, 42, 1417 (in Chinese).

(王兮, 张艳, 王剑波, 中国科学: 化学, 2012, 42, 1417.)

(j) Qing, F. L. Chin. J. Org. Chem. 2012, 32, 815 (in Chinese).

(卿风悀, 有机化学, 2012, 32, 815.)

(k) Lv, C. P.; Shen, Q. L.; Liu, D. Chin. J. Org. Chem. 2012, 32, 1380 (in Chinese).

(吕翠萍, 沈其龙, 刘丹, 有机化学, 2012, 32, 1380.)

(1) Pan, F.; Shi, Z. Acta Chim. Sinica 2012, 70, 1679 (in Chinese).

(潘菲, 施章杰, 化学学报, 2012, 70, 1679.)

(m) Yu, H. Z.; Su, S. Q.; Zhang, C.; Dang, Z. M. Chin. J. Org. Chem. 2013, 33, 1628 (in Chinese).

(于海珠, 苏圣钦, 张弛, 党智敏, 有机化学, 2013, 33, 1628.)

(n) Liu, T. F; Shen, Q, L. Eur. J. Org. Chem. 2012, 6679.

(o) Wang, G. Z.; He, X. P.; Dai, J. J.; Xu, H. J. Chin. J. Org. Chem. 2014, 34, 837 (in Chinese).

(王光祖, 赫侠平, 戴建军, 许华建, 有机化学, 2014, 34, 837.)

[2] (a) Swarts, F. Bull. Acad. R. Belg. 1892, 24, 309.

(b) Boswell, G. A., Jr.; Ripka, W. C.; Scribner, R. M.; Tullock, C. W. Org. React. 1974, $21,1$.

[3] (a) Dubinina, G. G.; Brennessel, W. W.; Miller, J. L.; Vicic, D. A. Organometallics 2008, 27, 3933.

(b) Wang, X.; Truesdale, L.; Yu, J.-Q. J. Am. Chem. Soc. 2010, 132, 3648 .

(c) Ye, Y.; Ball, N. D.; Kampf, J. W.; Sanford, M. S. J. Am. Chem. Soc. 2010, 132, 14682.

(d) Ye, Y.; Lee, S. H.; San ford, M. S. Org. Lett. 2011, 13, 5464.

(e) Parsons, A. T.; Senecal, T. D.; Buchwald, S. L. Angew. Chem., Int. Ed. 2012, 51, 2947.

(f) Herrmann, A. T.; Smith, L. L.; Zakarian, A. J. Am. Chem. Soc. 2012, 134, 6976.

(g) Iqbal, N.; Choi, S.; Kim, E.; Cho, E. J. J. Org. Chem. 2012, 77, 11383.

(h) Kazuyuki, S.; Masaaki, O.; Akira, A.; Itsumaro, K. Org. Lett. 2013, 23, 4359.

[4] (a) Zhang, S.-L.; Liu, L.; Fu, Y.; Guo, Q.-X. Organometallics 2007, 26, 4546 .

(b) Dubinina, G. G.; Furutachi, H.; Vicic, D. A. J. Am. Chem. Soc. 2008, 130, 8600 .

(c) Oishi, M.; Kondo, H.; Amii, H. Chem. Commun. 2009, 1909.

(d) Morimoto, H.; Tsubogo, T.; Litvinas, N. D.; Hartwig, J. F. Angew. Chem., Int. Ed. 2011, 50, 3793. 
(e) Zanardi, A.; Novikov, M. A.; Martin, E.; Benet-Buchholz, J.; Grushin, V. V. J. Am. Chem. Soc. 2011, 133, 20901.

(f) Tomashenko, O. A.; Escudero-Adan, E. C.; Belmonte, M. M.; Grushin, V. V. Angew. Chem., Int. Ed. 2011, 50, 7655.

(g) Zhang, C. P.; Wang, Z. L.; Chen, Q. Y.; Zhang, C. T.; Gu, Y. C.; Xiao, J. C. Angew. Chem., Int. Ed. 2011, 50, 1896.

(h) Weng, Z.; Lee, R.; Jia, W.; Yuan, Y.; Wang, W.; Feng, X.; Huang, K. W. Organometallics 2011, 30, 3229.

(i) Kondo, H.; Oishi, M.; Fujikawa, K.; Amii, H. Adv. Synth. Catal. 2011, 353, 1247.

(j) Knauber, T.; Arikan, F.; Roschenthaler, G.-V.; Goossen, L. J. Chem. Eur. J. 2011, 17, 2689.

(k) Duan, C.; Li, Y.; Chen, T.; Wang, H.; Zhang, R.; Jin, K.; Wang, X. Synlett 2011, 1713.

(1) Chen, M.; Buchwald, S. L. Angew. Chem., Int. Ed. 2013, 52, 11628.

(m) Hafner, A.; Braese, S. Adv. Synth. Catal. 2011, 353, 3044.

(n) Kawai, H.; Furukawa, T.; Nomura, Y.; Tokunaga, E.; Shibata, N. Org. Lett. 2011, 13, 3596.

(o) Schareina, T.; Wu, X.-F.; Zapf, A.; Cotté, A.; Gotta, M.; Beller, M. Top. Catal. 2012, 55, 426.

(p) Miyake, Y.; Ota, S.; Nishibayashi, Y. Chem.-Eur. J. 2012, 18, 13255.

(q) Zhao, T. S.; Szabo, K. J. Org. Lett. 2012, 14, 3966.

(r) Novak, P.; Lishchynskyi, A.; Grushin, V. V. J. Am. Chem. Soc. 2012, 134, 16167.

(s) Miyake, Y.; Ota, S.; Shibata, M.; Nakajima, K.; Nishibayashi, Y. Chem. Commum. 2013, 49, 7809.

(t) Larsson, J. M.; Pathipati, S. R.; Szabo, K. J. J. Org. Chem. 2013, 78,7330 .

(u) Ambler, B. R.; Altman, R. A. Org. Lett. 2013, 15, 5578.

[5] (a) Chu, L.; Qing, F.-L. Org. Lett. 2010, 12, 5060.

(b) Senecal, T. D.; Parsons, A. T.; Buchwald, S. L. J. Org. Chem. 2011, 76, 1174.

(c) Xu, J.; Luo, D. F.; Xiao, B.; Liu, Z. J.; Gong, T. J.; Fu, Y.; Liu, L. Chem. Commun. 2011, 47, 4300.

(d) Zhang, C. P.; Cai, J.; Zhou, C. B.; Wang, X. P.; Zheng, X.; Gu, Y. C.; Xiao, J. C. Chem. Commun. 2011, 47, 9516.

(e) Liu, T.; Shen, Q. Org. Lett. 2011, 13, 2342.

(f) Huang, Y.; Fang, X.; Lin, X.; Li, H.; He, W.; Huang, K.-W.; Yuan, Y.; Weng, Z. Tetrahedron 2012, 68, 9949.

(g) Ji, Y.; Brueckl, T.; Baxter, R. D.; Fujiwara, Y.; Seiple, I. B.; Su, S.; Blackmond, D. G.; Baran, P. S. Proc. Natl. Acad. Sci. U. S. A. 2011, $108,14411$.

(h) Ye, Y.; Kü nzi, S. A.; Sanford, M. S. Org. Lett. 2012, 14, 4979. (i) Litvinas, N. D.; Fier, P. S.; Hartwig, J. F. Angew. Chem., Int. Ed. 2012, $51,536$.

(j) Liu, T.; Shao, X.; Wu, Y.; Shen, Q. Angew. Chem., Int. Ed. 2012, 51, 555 .

(k) Novák, P.; Lishchynskyi, A.; Grushin, V. V. Angew. Chem., Int. Ed. 2012, 51, 7767.

(1) Khan, B. A.; Buba, A. E.; Goossen, L. J. Chem.-Eur. J. 2012, $18,1577$.

(m) Ye, Y.; Sanford, M. S. J. Am. Chem. Soc. 2012, 134, 9034.

(n) Xu, J.; Xiao, B.; Xie, C. Q.; Luo, D. F.; Liu, L.; Fu, Y. Angew. Chem., Int. Ed. 2012, 51, 12551.

(o) Zheng, H.; Huang, Y.; Wang, Z.; Li, H.; Huang, K.-W.; Yuan, Y.; Weng, Z. Tetrahedron Lett. 2012, 53, 6646.

(p) Li, Y.; Wu, L.; Neumann, H.; Beller, M. Chem. Commun. 2013, 49, 2628.

[6] (a) Chu, L.; Qing, F.-L. Org. Lett. 2010, 14, 2106.

(b) Xu, J.; Fu, Y.; Luo, D. F.; Jiang, Y. Y.; Xiao, B.; Liu, Z. J.; Gong, T. J.; Liu, L. J. Am. Chem. Soc. 2011, 133, 15300.

(c) Parsons, A. T.; Buchwald, S. L. Angew. Chem., Int. Ed. 2011, 50, 9120 .

(d) Wang, X.; Ye, Y.; Zhang, S.; Feng, J.; Xu, Y.; Zhang, Y.; Wang, J. J. Am. Chem. Soc. 2011, 133, 16410.

(e) Mitsudera, H.; Li, C.-J. Tetrahedron Lett. 2011, 52, 1898.

[7] (a) Shimizu, R.; Egami, H.; Nagi, T.; Chae, J.; Hamashima, Y.; Sodeoka, M. Tetrahedron Lett. 2010, 51, 5947.

(b) Chu, L.; Qing, F. L. J. Am. Chem. Soc. 2012, 134, 1298.

(c) Pair, E.; Monteiro, N.; Bouyssi, D.; Baudoin, O. Angew. Chem., Int. Ed. 2013, 52, 5346.

(d) Feng, C.; Loh, T.-P. Chem. Sci. 2012, 3, 3458.

(e) Ilchenko, N. O.; Janson, P. G.; Szabo, K. J. Chem. Commun. 2013, 49, 6614.

(f) Wang, X.; Ye, Y.; Ji, G.; Xu, Y.; Zhang, S.; Feng, J.; Zhang, Y.; Wang, J. Org. Lett. 2013, 15, 3730.

[8] (a) Chu, L.; Qing, F.-L. J. Am. Chem. Soc. 2010, 132, 7262.

(b) Weng, Z.; Li, H.; He, W.; Yao, L.-F.; Tan, J.; Chen, J.; Yuan, Y.; Huang, K.-W. Tetrahedron 2012, 68, 2527.

(c) Luo, D.-F.; Xu, J.; Fu, Y.; Guo, Q.-X. Tetrahedron Lett. 2012, $53,2769$.

(d) Zhang, K.; Qiu, X.-L.; Huang, Y.; Qing, F.-L. Eur. J. Org. Chem. 2012, 58.

[9] (a) Chen, Z.-M.; Bai, W.; Wang, S.-H.; Yang, B.-M.; Tu, Y.-Q.; Zhang, F.-M. Angew. Chem., Int. Ed. 2013, 52, 9781.

(b) Fang, Z.; Ning, Y.; Mi, P.; Liao, P.; Bi, X., Org. Lett. 2014, 16, 1522 . 